\title{
A critical role for NMDA receptors in parvalbumin interneurons for gamma rhythm induction and behavior
}

\author{
M Carlén ${ }^{1,2,9}$, K Meletis ${ }^{1,2,9}$, JH Siegle ${ }^{3}$, JA Cardin ${ }^{3,4}$, K Futai $^{1}$, D Vierling-Claassen ${ }^{3,5}$, C Rühlmann ${ }^{1}$, \\ SR Jones ${ }^{5}$, K Deisseroth ${ }^{6}$, M Sheng ${ }^{1}$, Cl Moore ${ }^{3}$ and L-H Tsai ${ }^{1,7,8}$ \\ ${ }^{1}$ Department of Brain and Cognitive Sciences, Picower Institute for Learning and Memory, MIT, Cambridge, MA, USA; \\ ${ }^{2}$ Department of Neuroscience, Karolinska Institutet, Stockholm, Sweden; ${ }^{3}$ McGovern Institute for Brain Research and \\ Department of Brain and Cognitive Sciences, MIT, Cambridge, MA, USA; ${ }^{4}$ Department of Neuroscience, University of \\ Pennsylvania, Philadelphia, PA, USA; ${ }^{5}$ Athinoula A. Martinos Center for Biomedical Imaging, Massachusetts General Hospital, \\ Harvard Medical School, Charlestown, MA, USA; ${ }^{6}$ Department of Bioengineering, Stanford University, Stanford, CA, USA; \\ ${ }^{7}$ Stanley Center for Psychiatric Research, Broad Institute of Harvard and Massachusetts Institute of Technology, Cambridge, \\ MA, USA and ${ }^{8}$ Howard Hughes Medical Institute, Cambridge, MA, USA
}

\begin{abstract}
Synchronous recruitment of fast-spiking (FS) parvalbumin (PV) interneurons generates gamma oscillations, rhythms that emerge during performance of cognitive tasks. Administration of $\mathrm{N}$-methyl-D-aspartate (NMDA) receptor antagonists alters gamma rhythms, and can induce cognitive as well as psychosis-like symptoms in humans. The disruption of NMDA receptor (NMDAR) signaling specifically in FS PV interneurons is therefore hypothesized to give rise to neural network dysfunction that could underlie these symptoms. To address the connection between NMDAR activity, FS PV interneurons, gamma oscillations and behavior, we generated mice lacking NMDAR neurotransmission only in PV cells (PV-Cre/NR1f/f mice). Here, we show that mutant mice exhibit enhanced baseline cortical gamma rhythms, impaired gamma rhythm induction after optogenetic drive of PV interneurons and reduced sensitivity to the effects of NMDAR antagonists on gamma oscillations and stereotypies. Mutant mice show largely normal behaviors except for selective cognitive impairments, including deficits in habituation, working memory and associative learning. Our results provide evidence for the critical role of NMDAR in PV interneurons for expression of normal gamma rhythms and specific cognitive behaviors.

Molecular Psychiatry (2012) 17, 537-548; doi:10.1038/mp.2011.31; published online 5 April 2011
\end{abstract}

Keywords: parvalbumin; interneurons; gamma; NMDAR; optogenetics; oscillations

\section{Introduction}

Fast-spiking (FS) parvalbumin (PV) interneurons are gamma-aminobutyric acid (GABA)-ergic cells that express the $\mathrm{Ca}^{2+}$-binding protein $\mathrm{PV}$ and receive $N$-methyl-D-aspartate (NMDA)-dependent excitatory input from pyramidal cells. ${ }^{1}$ FS-PV interneurons regulate the activity of neural networks through GABA-ergic inhibition of local excitatory neurons, and synchronous activity of FS interneurons generates gamma oscillations $(30-80 \mathrm{~Hz}) .^{2-6}$ Normal gamma oscillations are correlated with performance of a

Correspondence: Dr M Carlén, Department of Neuroscience, Karolinska Institutet, Retzius väg 8, 17177 Stockholm, Sweden or Dr CI Moore, McGovern Institute for Brain Research, Massachusetts Institute of Technology, 77 Massachusetts Avenue, Cambridge, MA 02139, USA or Dr L-H Tsai, Picower Institute for Leaning and Memory, Massachusetts Institute of Technology, 77 Massachusetts Avenue, Cambridge, MA 02139, USA.

E-mail: marie.carlen@ki.se or cim@mit.edu or lhtsai@mit.edu ${ }^{9}$ These authors contributed equally to this work.

Received 26 September 2010; revised 19 January 2011; accepted 24 February 2011; published online 5 April 2011 variety of cognitive tasks, including the allocation of attention and working memory. ${ }^{7}$ Consistent with the importance of gamma oscillations, and with the more general requirement of appropriate inhibition in neural circuits, FS-PV interneurons have been proposed to underlie the cognitive disturbances associated with psychiatric disorders. ${ }^{8}$

The NMDA receptor (NMDAR) has also been directly implicated in the emergence of gamma rhythms, as NMDAR antagonists can disrupt ${ }^{9}$ or potentiate, ${ }^{10}$ gamma rhythms in slices and in vivo. ${ }^{11-15}$ This aspect of the glutamatergic system is also implicated in cognitive disturbances by the psychotomimetic effects of NMDAR antagonists, ${ }^{16,17}$ which recapitulate core features of schizophrenia, most notably cognitive deficits in planning, attention, learning and memory. ${ }^{18,19}$ Further, schizophrenic patients display aberrant induction of gamma oscillations during cognitive tasks. ${ }^{7,20,21}$

These intersecting lines of evidence strongly implicate PV interneurons and NMDAR in the expression of gamma rhythms in normal cognitive 
function and in disease. To directly test the impact of NMDAR function in PV interneurons on gamma oscillation expression and behavior, we generated mice lacking NMDAR only in PV neurons. We then probed the resting-state expression of gamma oscillations, and their induction through optogenetic and pharmacological means. We also measured a variety of behaviors ranging from sensorimotor gating to cognitive dimensions such as working memory. We found that selective deletion of NMDAR from PV cells leads to an enhanced resting-state expression of gamma oscillations and a deficit in gamma induction. Further, we found a selective pattern of behavioral disturbance that spares several metrics but impairs expression of habituation, working memory and other learning indices. These data strongly support the role of NMDAR on PV cells in normal gamma functionality in the in vivo mammalian neocortex, and indicate a specific role for it in selective aspects of learning and memory.

\section{Subjects and methods}

Animals

All procedures were conducted in accordance with the National Institutes of Health guidelines and with the approval of the Committee on Animal Care at MIT, Cambridge, MA, USA. We generated PV-Cre/NR1f/f mice by crossing of PV-Cre mice ${ }^{22}$ with mice carrying 'floxed' NR1 alleles. ${ }^{23}$ In awake electrophysiology and behavior tests, male PV-Cre/NR1f/f mice and littermate male controls (NR1f/f) were used. For slice electrophysiology and optogenetic experiments in anesthetized animals, age-matched male PV-Cre mice were used as controls.

\section{Immunohistochemistry}

Free-floating sections $(30 \mu \mathrm{m})$ were prepared and immunostained. ${ }^{4}$ The following primary antibodies were used: PV PVG-214 (Swant, Bellinzona, Switzerland; 1:2000), enhanced yellow fluorescent protein (EYFP) (GFP-1020 Aves, Tigard, OR, USA; 1:500). Antibody staining was revealed using speciesspecific fluorophore-conjugated secondary antibodies (Cy5 from Jackson, West Grove, PA, USA, Alexa 488 from Molecular Probes, San Diego, CA, USA).

\section{Quantification}

Cre recombination was quantified in PV-Cre mice crossed to the R26R-EYFP Cre reporter mouse line. ${ }^{24}$ Free-floating sections stained with antibodies against PV and EYFP were used. For quantification of recombination in S1, every PV cell was counted and scored for co-labeling with EYFP in $1190.30 \times 1190.30 \times 30 \mu \mathrm{m}$ images including all six cortical layers. For quantification of recombination in hippocampus every PV cell in dentate gyrus, CA1, CA2 and CA3 was counted and scored for co-labeling with EYFP.

For quantification of the number and distribution of PV cells in S1 in NR1f/f and PV-Cre/NR1f/f mice and every PV cell was counted as describe above and assigned to layers $2 / 3$ or $4-6$ based on its position in relation to the layer 4 barrels.

Slice electrophysiology

AAV DIO channelrhodopsin-2 (ChR2)-mCherry ${ }^{4}$ was injected into hippocampus of 5- to 7-week-old PV-Cre and PV-Cre/NR1f/f mice. At 7-10 days after viral transduction, transverse hippocampal slices (400 $\mu \mathrm{m}$ thickness) were prepared as described. ${ }^{25,26}$ The tungsten bipolar electrode (FHC, Bowdoin, ME, USA) was placed in the stratum radiatum or oriens and the Schaffer collateral/commissural fibers were stimulated at $0.1 \mathrm{~Hz}$. Picrotoxin $(0.15 \mathrm{~mm}$, Sigma, St Louis, MO, USA) was dissolved in artificial cerebrospinal fluid (aCSF) to block $\mathrm{GABA}_{\mathrm{A}}$ receptor-mediated synaptic transmission for whole-cell patch clamp recordings. $\alpha$-Amino-3-hydroxy-5-methyl-4-isoxazolepropionic acid receptor-mediated excitatory postsynaptic current (EPSCs) were recorded at $-70 \mathrm{mV}$ and NMDARmediated EPSCs were recorded at $+40 \mathrm{mV}$ with the same stimulus strength in the presence of NBQX (0.005 mM, Tocris, Ellisville, MO, USA).

\section{Anesthetized electrophysiology}

AAV DIO ChR2-mCherry was injected into barrel cortex of adult (8-12 weeks old) PV-Cre or PV-Cre/ NR1f/f mice as described earlier. ${ }^{4}$ Electrophysiological in vivo recordings were performed 1-3 weeks after viral injections. Extracellular single-unit and local field potential (LFP) recordings were made with tetrodes or stereotrodes. Stimulus control and data acquisition was performed using software custom written in LabView (National Instruments, Austin, TX, USA) and Matlab (The Mathworks, Natick, MA, USA) by Ulf Knoblich.

Light stimulation was generated by a $473 \mathrm{~nm}$ laser and light pulses were given via a $200 \mu \mathrm{m}$ diameter, unjacketed optical fiber at the cortical surface 75-200 $\mu \mathrm{m}$ from the recording electrodes.

Unit and local field potential analysis used software custom written in Igor Pro (Wavemetrics, Portland, OR, USA) by JAC. Spontaneous anesthetized LFP measurements were made during periods with no light stimulation. For each light stimulation frequency, we measured relative power ${ }^{27-29}$ in an $8 \mathrm{~Hz}$ band centered on that frequency. Relative power was used to account for differences in LFP amplitude between recording sites and between electrodes. Relative power was calculated by measuring the ratio of power within the band of interest to total power (1$100 \mathrm{~Hz}$ ) in the power spectrum of the unfiltered LFP (for details see Supplementary Information; extended Materials and Methods). For each recording site, we measured power from 10 to $30 \mathrm{LFP}$ traces under each condition. Example power spectra are population averages. We also measured the power ratio:

$$
P_{\text {light }} / P_{\text {baseline }} \text {, }
$$

where $P_{\text {light }}$ is the relative power in a frequency band in the presence of light stimulation and $P_{\text {baseline }}$ is the power in that band in the absence of light stimulation. 
Spike waveforms of regular spiking (RS) and FS cells were characterized. In each case, FS measurements were made from cells expressing ChR2 and driven by light pulses and RS measurements were made from non-driven, spontaneously active cells. Statistical significance was assessed with the Mann-Whitney test. All numbers are given as mean \pm s.e.m., except where otherwise noted.

\section{Awake electrophysiology}

Eight NR1f/f control and eight PV-Cre/NR1f/f mice (8-10 weeks) were used for awake electrophysiology. Teflon-coated tungsten electrodes (impedance of $100 \mathrm{k} \Omega$ ) were implanted: two electrodes were placed bilaterally in primary somatosensory (barrel) cortices $1.5 \mathrm{~mm}$ posterior to bregma and $3.5 \mathrm{~mm}$ from the midline. In each hemisphere, a signal electrode was implanted $0.5 \mathrm{~mm}$ below the cortical surface and a reference electrode was implanted $1.75 \mathrm{~mm}$ below the cortical surface. A stainless steel screw over right posterior parietal cortex served as ground. Recording sessions took place in an empty box to which animals had not been previously exposed. After a 5-10-min habituation period, $20 \mathrm{~min}$ of baseline data were recorded for each animal. After the baseline period, four NR1f/f control and four PV-Cre/NR1f/f mice were injected intraperitoneally with $0.5 \mathrm{mg} \mathrm{kg}^{-1}$ of MK-801 and four NR1f/f control and four PV-Cre/NR1f/f mice were injected intraperitoneally with saline and another $40 \mathrm{~min}$ of data were recorded. The behavioral state of all animals was scored by an observer blind to the genotype, and the sessions for ten of the animals were monitored with a video camera. All analysis was performed offline in Matlab. Normalized relative power indicates power normalized by both total power and by relative power during the baseline period, to highlight pre/post changes. Power change is the logarithm of the normalized relative power, which facilitates comparisons across frequency bands. Statistical significance was assessed with the MannWhitney test (between-group comparisons) and the Wilcoxon signed-rank test (within-group comparisons).

\section{Mouse behavior}

All behavioral tests were performed blind for the genotype of the mice. Male PV-Cre/NR1f/f mice and littermate male controls (NR1f/f) were used for all behavioral tests. Behavioral parameters were analyzed by $t$-tests for the two genotypes, except for otherwise noted, and corrected for multiple comparisons. $P<0.05$ was considered significant. All results are presented as mean \pm s.e.m.

\section{Open field analysis}

Activity in a novel open field was measured in monitors with sets of 16 light beam arrays. During 60 min the hardware detected beams broken by the animal, with the software determining the location and activity of the animal. For the pharmacological treatment, one set of mice was first monitored in the open field for $30 \mathrm{~min}$. Directly thereafter MK-801

(M107; Sigma) (0.2 or $\left.0.3 \mathrm{mg} \mathrm{kg}^{-1}\right)$ was injected intraperitoneally and the mice were monitored for additionally $60 \mathrm{~min}$, in the same boxes as before.

\section{Acoustic startle and prepulse inhibition}

For testing of sensorimotor gating, startle response and prepulse inhibition (PPI) were determined using the Startle Monitor System (SM100; Hamilton Kinder, San Diego, CA, USA). The animals were habituated to the experimental equipment for two days. Day 3, the PPI testing day, each animal was exposed to $65 \mathrm{~dB}$ ambient noise for $5 \mathrm{~min}$ followed by the testing session. The PPI paradigm consisted of trials with presentation of a startle stimulus alone and trials where a prepulse of varying intensity preceded the startle stimulus by $100 \mathrm{~ms}$. Trials were presented in blocks, with each block consisting of one startle stimulus alone trial, each of the prepulse-pulse stimulus trials, and a no stimulus trial, in a fixed pseudo randomized order. A total of six blocks were presented in a session and corresponding responses were averaged for each mouse and trial separately.

\section{Habituation}

Habituation of the startle reflex was assessed by comparing the mean startle amplitude of four pulses of a $120 \mathrm{~dB}$ white noise stimulus presented at the beginning and the end of the startle session using the equation (100-(mean startle block 2/mean startle block 1) × 100)).

\section{T-maze test}

For testing of working memory, a modified T-maze, the discrete paired-trial variable-delay alternation task, was used. ${ }^{30}$

\section{Contextual and cued fear conditioning}

Context-dependent fear conditioning: the animals were placed in a novel conditioning chamber for $3 \mathrm{~min}$ where they were exposed to a foot shock. After $24 \mathrm{~h}$, animals were returned to the same chamber and contextual memory was assessed as freezing during a 3-min test period. Tone-dependent (cued) fear conditioning: the animals were placed in the conditioning chamber for $3 \mathrm{~min}$ after which a 20-s tone (auditory cue) followed which co-terminated with a foot shock. After $24 \mathrm{~h}$, animals were returned and cued fear learning was assessed. Before placement of the animals in the conditioning chamber, the environment in the chamber had been changed (visual, tactile and olfactory cues) to present the animals with a new context for the test.

\section{Water maze}

The spatial reference task was a water maze task performed in a circular tank (diameter $1.8 \mathrm{~m}$ ) filled with opaque water. A fixed platform $(10 \mathrm{~cm}$ diameter $)$ was hidden below the water's surface in the center of the target quadrant. After training for several days, the platform was removed (probe trial). Reversal training started the day after the probe trial, followed by a probe trial for the new target quadrant. 


\section{Results}

\section{Genetic ablation of NMDAR signaling in $P V$} interneurons

Previous mouse models of NMDAR hypofunction have targeted all neuronal classes, resulting in severe behavioral abnormalities. ${ }^{31,32}$ To directly address the function of NMDAR specifically in PV interneurons in network function and cognitive behavior, we created mice lacking the NMDAR subunit NR1 only in PV-expressing cells. Mice with Cre recombinase expression in PV cells (PV-Cre mice) ${ }^{22}$ were crossed with mice carrying floxed alleles of the NR1 subunit (NR1f/f mice). ${ }^{23}$

\section{Characterization of $P V$-Cre/NR1f/f mice}

PV-Cre/NR1f/f mice were viable, developed normally and did not exhibit growth abnormalities or other gross anatomical changes (data not shown). Credependent recombination from the PV locus was specific to PV-expressing cells and followed the postnatal onset of PV expression ${ }^{33}$ (Figure 1a). PVCre-driven recombination in somatosensory cortex and hippocampus was detected at postnatal day 13 (P13) with increased recombination at 29 days (P29) and almost complete recombination at 8 weeks (Figure 1a, Supplementary Figure 1). Whole-cell recordings in hippocampal slices in vitro confirmed the functional loss of NMDAR currents in PV cells in PV-Cre/NR1f/f mice (five cells in four PV-Cre/NR1f/f mice, seven cells in five control mice, $P=0.03$, unpaired $t$-test; Figures $1 \mathrm{~b}$ and $\mathrm{c}$ ). We found the cortical architecture (layers and barrels in somatosensory cortex), migration and differentiation of $\mathrm{PV}$ interneurons to be normal in PV-Cre/NR1f/f mice (Figures $1 \mathrm{~d}$ and e; $P=0.65$ for layers $2 / 3$ and $P=0.53$ for layers 4-6, two-tailed unpaired $t$-test; $n=3$ per genotype).

\section{Spontaneous and induced gamma oscillations are altered in PV-Cre/NR1f/f mice}

We have previously described that activation of FS-PV interneurons enhances gamma oscillations in neocortex in vivo, ${ }^{4}$ in agreement with substantial correlative and theoretical previous evidence. ${ }^{34,35}$ Somatosensory cortex represents a local circuit with well-described anatomy and function, arguably being the most widely employed model of neocortical function in the rodent. FS-PV interneurons are local circuit neurons, and recent studies in somatosensory cortex have provided substantial progress for our understanding of FS behavior in gamma rhythm generation in general cortical network functions in vivo. ${ }^{4,6}$ There is also a substantial previous work measuring receptive fields of FS interneurons in this specific cortical area. ${ }^{36-38}$ Recently, it was shown that NMDAR antagonists increase the power of basal gamma oscillations in rodents. ${ }^{13}$ Abnormal gamma was found to be ongoing simultaneously all over the
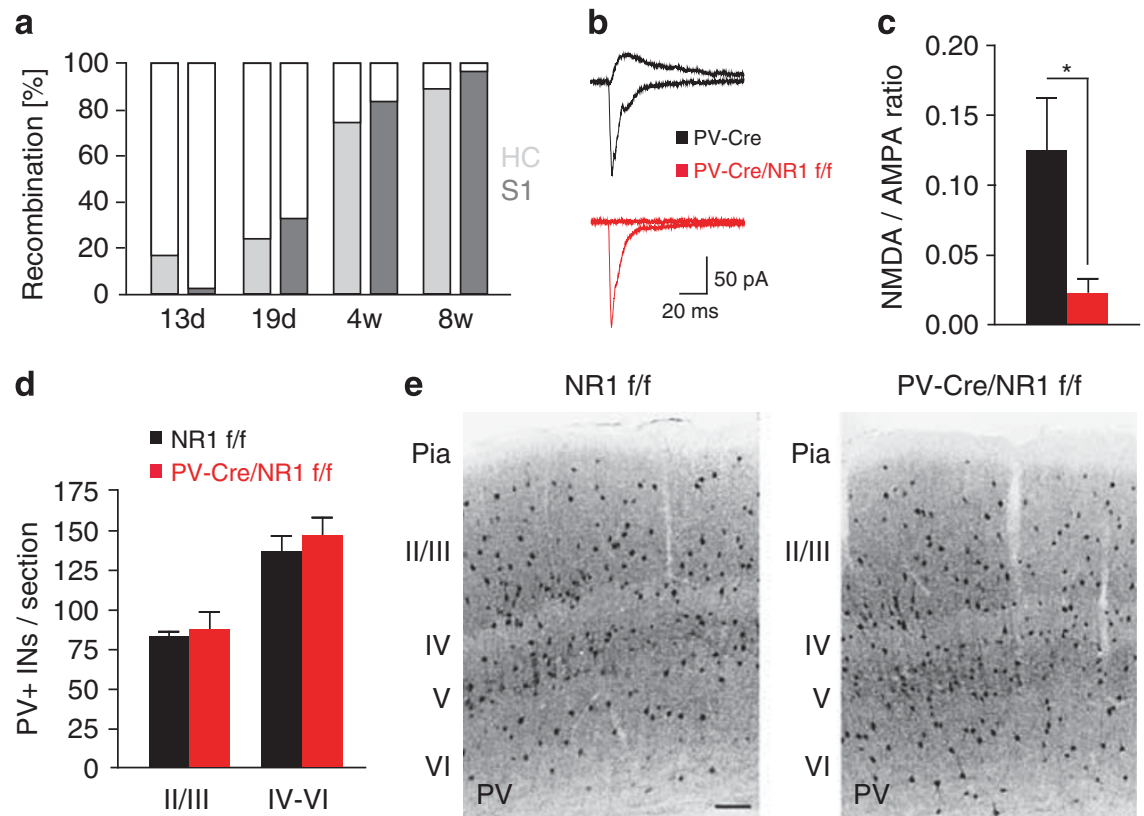

Figure 1 Genetic ablation of NMDA receptor (NMDAR) specifically in parvalbumin (PV) interneurons. (a) Quantification of recombination from the PV locus in somatosensory cortex and hippocampus at different time points. (b, c) NMDAR-mediated synaptic transmission in PV interneurons is abolished in PV-Cre/NR1f/f mice. (b) Sample EPSC traces mediated by the AMPAR (downward) and NMDAR (upward) from a control PV-Cre mouse and a PV-Cre/NR1f/f mouse. (c) NMDAR EPSC/ AMPAR EPSC ratio in control PV-Cre and PV-Cre/NR1f/f mice. (d) Distribution of PV interneurons in NR1f/f and PV-Cre/ NR1f/f mice, respectively, at 11 weeks. (e) Immunohistochemistry for PV interneurons in somatosensory cortex of an adult NR1f/f and PV-Cre/NR1f/f mouse, respectively. ${ }^{*} P<0.05$; error bars, mean \pm s.e.m. Scale bar: (e) $200 \mu \mathrm{m}$. See also Supplementary Figure 1. AMPAR, $\alpha$-amino-3-hydroxy-5-methyl-4-isoxazolepropionic acid receptor; EPSC, excitatory postsynaptic current. 
cerebral cortex (prefrontal, frontal, parietal and occipital areas) in free-moving, sedated and anesthetized animals. ${ }^{13}$ The dysfunction in information processing found in schizophrenic patients is not limited to high-order association cortex, such as prefrontal cortex, but also influences sensory cortex. ${ }^{39}$
To identify potential changes in baseline cortical rhythms, we recorded spontaneous LFPs in layers 2/3 and 4 of primary somatosensory (barrel) cortex of anesthetized PV-Cre/NR1f/f mice $(n=8$ sites in five control mice and 10 sites in six PV-Cre/NR1f/f mice; Figures $2 \mathrm{a}$ and $\mathrm{b}$ ). We found a significant decrease in a

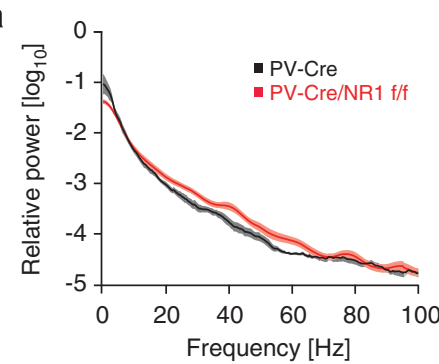

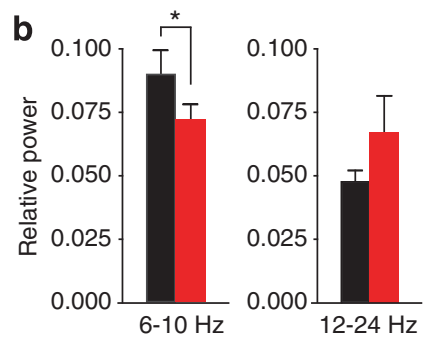
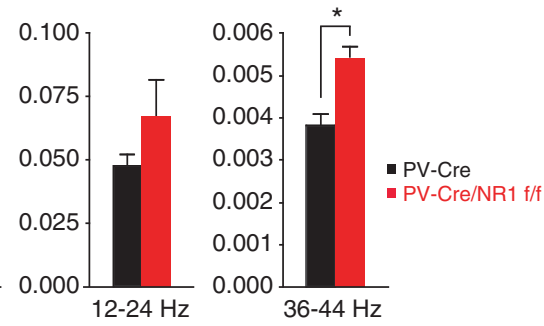

C

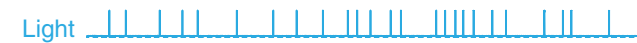

PV-Cre

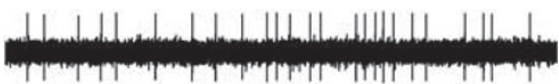

PV-Cre/NR1 f/f

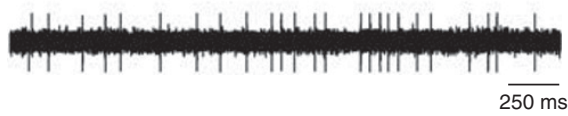

d

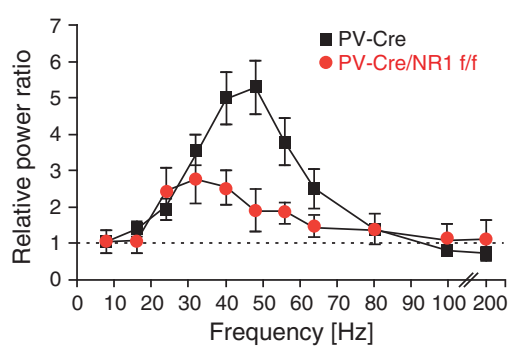

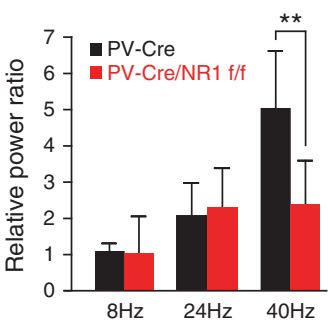

f $\quad \mathrm{PV}-\mathrm{Cre} / \mathrm{NR} 1 \mathrm{f} / \mathrm{f}$ Baseline

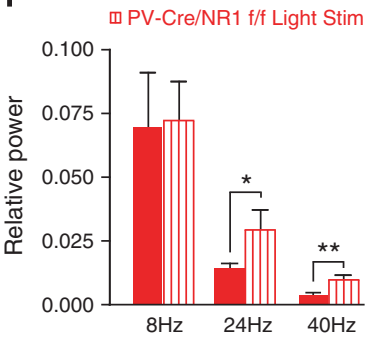

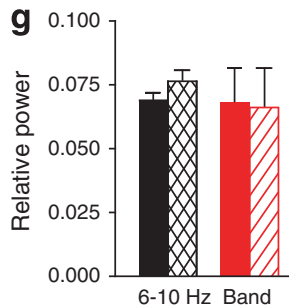
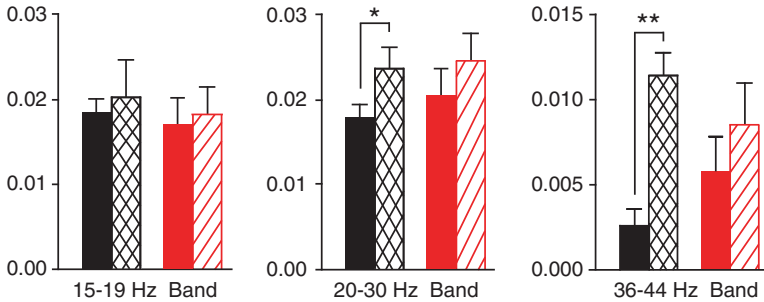

- PV-Cre Baseline XV-Cre Broadband Stim

$\square$ PV-Cre/NR1 f/f Broadband Stim

Figure 2 Spontaneous and induced cortical gamma oscillations require NMDA receptor (NMDAR) in parvalbumin (PV) interneurons. (a, b, d-g) Local field potential (LFP) activity in anesthetized control (black) and PV-Cre/NR1f/f (red) mice. (a, b) Spontaneous LFP activity. (a) Relative power from 1-100 Hz in control and PV-Cre/NR1f/f mice. (b) Relative LFP power in the $6-10,12-24$ and $36-44 \mathrm{~Hz}$ frequency bands. (c) Single-unit recordings during optogenetic activation of FS-PV + interneurons. Middle trace: control mice, lower trace: PV-Cre/NR1f/f mice. In each case, a series of $1 \mathrm{~ms}$ light pulses (blue trace) was given in a random pattern drawn from a broadband distribution (5-200 Hz). In both cases, each light pulse evoked a single spike from the cell. Both cells followed the light stimulus with a high degree of reliability. (d-g) Optogenetic activation of FS-PV interneurons in somatosensory cortex in control and PV-Cre/NR1f/f mice. (d) Mean power ratio in each LFP frequency band in response to light activation of channelrhodopsin-2 (ChR2)-expressing FS-PV interneurons at varying frequencies. PV-Cre/NR1f/f mice generate significantly less $30-60 \mathrm{~Hz}$ oscillations (gamma) than control mice. (e) Comparison of the effect of activating FS-PV interneurons in the control and PV-Cre/NR1f/f mice at 8, 24, and $40 \mathrm{~Hz}$ on relative LFP power in those frequency bands. (f) Relative LFP power in the 8, 24 and $40 \mathrm{~Hz}$ frequency bands in the PV-Cre/NR1f/f mice during spontaneous (solid bars) and light-evoked (striped bars) activity. (g) Relative power in the 610, 15-19, 20-30 and 36-44 Hz frequency bands in response to broadband light stimulation in control and PV-Cre/NR1f/f mice. ${ }^{*} P<0.05,{ }^{*} P<0.01$; error bars, mean \pm s.e.m. 
relative power ${ }^{27-29}$ in the $6-10 \mathrm{~Hz}$ theta frequency band $(P<0.05$; Figure $2 \mathrm{~b})$ and a significant increase in relative power in the $36-44 \mathrm{~Hz}$ gamma frequency band $(P<0.05$; Figure 2b) in $P V-C r e / N R 1 f / f$ mice compared with control mice, whereas LFP activity at slightly lower frequencies in the $12-24 \mathrm{~Hz}$ betafrequency band was not significantly altered in baseline conditions in PV-Cre/NR1f/f mice (Figure 2b). RS, putative excitatory neurons in PV-Cre/NR1f/f mice showed a significantly higher spontaneous firing rate $(n=16$ cells; $0.49 \pm 0.09 \mathrm{~Hz})$ than did RS cells in control mice $(n=18$ cells; $0.25 \pm 0.06 \mathrm{~Hz}$; Mann-Whitney test; $P<0.05$; data not shown).

We next tested the role of NMDAR in FS-PV interneurons for the induction of gamma oscillations in cortical networks by direct recruitment of FS-PV interneurons through the specific expression of the light-activated channel ChR2, as described previously. ${ }^{4}$ We drove ChR2-expressing FS-PV cells at a range of frequencies $(8-200 \mathrm{~Hz})$ in bouts of $3 \mathrm{~s}$ of 1 ms light pulses $(n=7$ sites in five control mice and 12 sites in six PV-Cre/NR1f/f mice; Figures 2c-f). Across our sample, FS-PV interneurons in control and PV-Cre/NR1f/f mice were driven with high reliability by light pulses (Figure 2c). Light pulses in the gamma range resulted in significant amplification of relative LFP power at those frequencies in control mice $(P<0.01$; Figure 2d). Driving FS-PV interneurons lacking NMDAR revealed a specific disruption in the ability of the local network to induce gamma oscillations, resulting in a significantly reduced gamma activity enhancement compared with control mice (Figure 2d). The specificity of this disruption to the gamma band in PV-Cre/NR1f/f mice was highlighted when FS-PV cells were driven at 8, 24 and $40 \mathrm{~Hz}$, respectively (Figure 2e). The oscillatory disruption was not complete as optogenetic stimulation at 24 or $40 \mathrm{~Hz}$ each induced significantly increased activity compared with baseline conditions in PVCre/NR1f/f mice $(P<0.05$; Figure 2f).

We used randomly patterned light stimulation (broadband stimulation ${ }^{4}$ ) of FS-PV interneurons to probe the emergent properties of the local cortical circuit. This paradigm, in agreement with periodic light stimulation, evoked a significant increase in 20$30 \mathrm{~Hz}$ and $36-44 \mathrm{~Hz}$ activity in control $(P<0.05)$ but not $\mathrm{PV}-\mathrm{Cre} / \mathrm{NR} 1 / \mathrm{f} / \mathrm{f}$ mice $(P>0.05 ; n=7$ sites in five control and 10 sites in six PV-Cre/NR1f/f mice; Figure 2g).

To probe the impact of NMDAR deletion on recruitment of FS-PV interneurons, we analyzed spike latency and variance in spike timing following optical stimulation in PV-Cre/NR1f/f mice. We found a diminished spike synchronization of FS units in response to light activation (Supplementary Figures 2b, c and g) compared with control mice (Supplementary Figures 2e-g) with significantly increased spike latency and variance in spike timing. As shown in this example, we observed reduced inhibition of RS neurons early in the gamma cycle (4-10 ms after light pulse; Supplementary Figures 2a and c) as well as diminished entrainment of the RS neurons at the end of the cycle (around $20 \mathrm{~ms}$ after light pulse; Supplementary Figures 2a and c) compared with control mice (Supplementary Figures 2d and f).

Computational modeling, described below, suggests that reduced synchronization of the FS-PV population results in higher gamma band activity during baseline conditions as well as a reduced ability to recruit and synchronize the local network during higher levels of excitatory activity (Supplementary Figure 3a).

To test whether deficits in cortical network activity were also present in awake behaving animals, we performed LFP recordings in layers $2 / 3$ and 4 of somatosensory cortex in freely moving PV-Cre/NR1f/f $(n=7)$ or control mice $(n=7)$ during behavior in a novel environment. We observed prominent gammaband oscillations in both genotypes (Figure 3a). During a baseline recording period, PV-Cre/NR1f/f mice exhibited a nonsignificant trend toward elevated spontaneous LFP in the $30-80 \mathrm{~Hz}$ gamma range independent of specific behaviors such as grooming or active exploration, compared with control mice (Figure 3b). PV-Cre/NR1f/f mice showed significantly longer mean event durations for spontaneous gamma oscillations compared with control mice $(P<0.005)$ without changes in the number of gamma events (Figure 3c).

To explore the role of NMDAR in PV interneurons on evoked gamma activity in the awake state, we challenged PV-Cre/NR1f/f mice with an acute administration of the non-competitive NMDAR antagonist MK-801 (0.5 mg kg-1; intraperitoneal; Figures 3d-g) or saline (data not shown). In agreement with previous findings, ${ }^{14}$ control mice acutely displayed a significant increase in LFP in the $30-50 \mathrm{~Hz}$ gamma range (post1, 5-15 min after MK-801 administration; $P<0.05$, Wilcoxon signed-rank test; Figure 3e). PVCre/NR1f/f mice, in contrast, displayed a significant reduction in gamma-band activity after NMDAR antagonist treatment (Figure 3e). Control mice and PV-Cre/NR1f/f mice displayed a significant induction in the $6-10 \mathrm{~Hz}$ frequency band relative power at later time points (post2, 25-35 min after MK-801 administration; Supplementary Figures $4 \mathrm{a}$ and b). In the 12$24 \mathrm{~Hz}$ band, the relative power remained unchanged in control animals during the same time frame, but was significantly reduced in PV-Cre/NR1f/f animals (Supplementary Figures 4c and d). Saline treatment did not induce any significant changes in locomotor behavior or relative power in any frequency band between genotypes throughout the duration of the recording sessions (data not shown).

To interpret the findings relative to established theories of gamma emergence, we conducted simulations using a modified form of an established neural circuit model. ${ }^{40}$ We simulated NMDAR dysfunction in FS-PV interneurons and found emergence of higher frequency oscillations in baseline activity, analogous to the experimental data (Supplementary Figure 3a and Supplementary Text; Modeling results). 
a

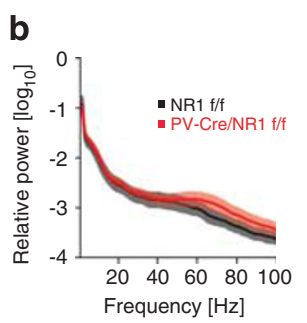

f
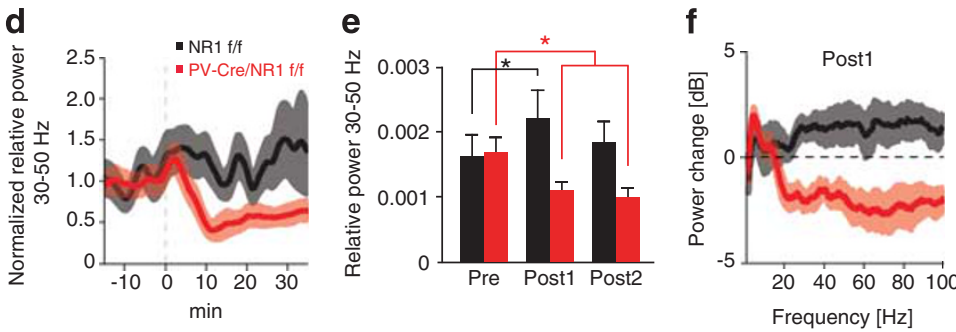

g

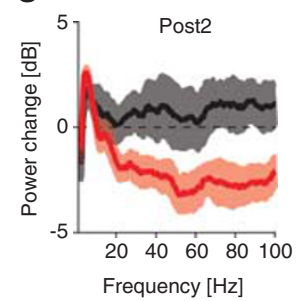

Figure 3 Baseline cortical oscillations and NMDA receptor (NMDAR) antagonist induced gamma rhythms in awake behaving animals. (a-g) Local field potential (LFP) activity in somatosensory cortex in awake control and parvalbumin (PV)Cre/NR1f/f mice. (a) Examples of single-trial gamma activity from a PV-Cre/NR1f/f and control mouse. Thin lines: LFP filtered between 5-300 Hz; thick lines: LFP filtered in the $30-50 \mathrm{~Hz}$ gamma frequency range. (b, c) Awake baseline activity. (b) Mean power spectra 1-100 Hz. Lighter regions indicate s.e.m. (c) Characteristics of 30-50 Hz gamma events. Mean event duration is significantly increased in freely moving PV-Cre/NR1f/f mice, whereas number of events are not. (d-g) NMDAR antagonist (MK-801) challenge. (d, e) Average relative power in the $30-50 \mathrm{~Hz}$ gamma frequency band $15 \mathrm{~min}$ before administration of MK-801 (dashed line), to 35 min after. Lighter regions indicate s.e.m. Administration of MK-801 gives a significant increase in relative gamma power in control mice and a significant reduction in relative gamma power in PV-Cre/ NR1f/f mice. (f) Average power changes (dB) between pre and post1. Lighter regions indicate s.e.m. (g) Average power changes $(\mathrm{dB})$ between pre and post2. Pre, 5-15 min before MK-801; post1, 5-15 min after MK-801; post2, 25-35 min after MK801. ${ }^{*} P<0.05$; error bars, mean \pm s.e.m. See also Supplementary Figures 3 and 4.

We also observed a significant reduction in gammaband activity following simulation of pharmacological NMDAR inhibition in a PV-Cre/NR1f/f network (Supplementary Figure 3b and Supplementary Text; Modeling results). In addition, we found an increase in the latency and variation of the FS-PV response to simulated light drive that may be responsible for the smaller enhancement of gamma power in this condition (Supplementary Figure 3c and Supplementary Text; Modeling results).

\section{Behavioral effects of NMDAR deletion in PV cells}

We characterized the PV-Cre/NR1f/f mice in a series of paradigms to assess general locomotor and exploratory behaviors as well as cognitive tasks evaluating learning and memory. The PV-Cre/NR1f/f mice and their littermate controls (NR1f/f) were introduced into an automated novel open field environment at an age of 7 weeks ( $n=11$ per genotype). We did not observe any consistent behavioral differences between PV-Cre/NR1f/f and control mice over the entire $60 \mathrm{~min}$ trial or in any 5 min block after analysis of 18 different parameters, including total distance (Figure 4a), locomotion, stereotypy and center/margin time (data not shown).

We questioned whether PV-Cre/NR1f/f mice at later stages develop behavioral abnormalities in the open field and therefore assessed mice of 11-12 weeks of age ( $n=9$ per genotype). We found a nonsignificant trend toward a decrease in total distance traveled over the 60-min period for PV-Cre/NR1f/f mice compared with control mice $(P>0.05$; Mann-Whitney test; Figure 4b). Analysis of time spent in the center (Figure 4c) or margin of the open field, vertical movements (data not shown) or stereotypy counts (Figure 4d) did not reveal differences between the genotypes over the 60-min period.

Our electrophysiological findings indicate that the PV-Cre/NR1f/f mice have a selective deficit in gamma emergence following optogenetic drive of FS-PV interneurons or pharmacological NMDAR inhibition. To investigate potential behavioral differences in response to acute NMDAR inhibition, we exposed naïve PV-Cre/NR1f/f and control mice $(n=11$ per genotype) to the open field for $30 \mathrm{~min}$ and then challenged them with administration of the NMDAR antagonist MK-801 at a low dose $\left(0.3 \mathrm{mg} \mathrm{kg}^{-1}\right)$ followed by continued exposure to the open field for $60 \mathrm{~min}$. Control mice responded over time to MK-801 administration with increased horizontal activity and induction of stereotypies as previously reported ${ }^{31}$ (Figures $4 \mathrm{e}$ and f). In contrast, PV-Cre/NR1f/f mice showed a markedly reduced sensitivity to MK801 (Figures 4e and f), consistent with our electrophysiological findings that PV interneurons are an important target for the non-competitive NMDAR antagonists.

PPI is associated with deficiencies in sensory information filtering and has been well characterized in schizophrenia. ${ }^{41,42}$ PPI deficits have repetitively been found in schizophrenia patients and their 

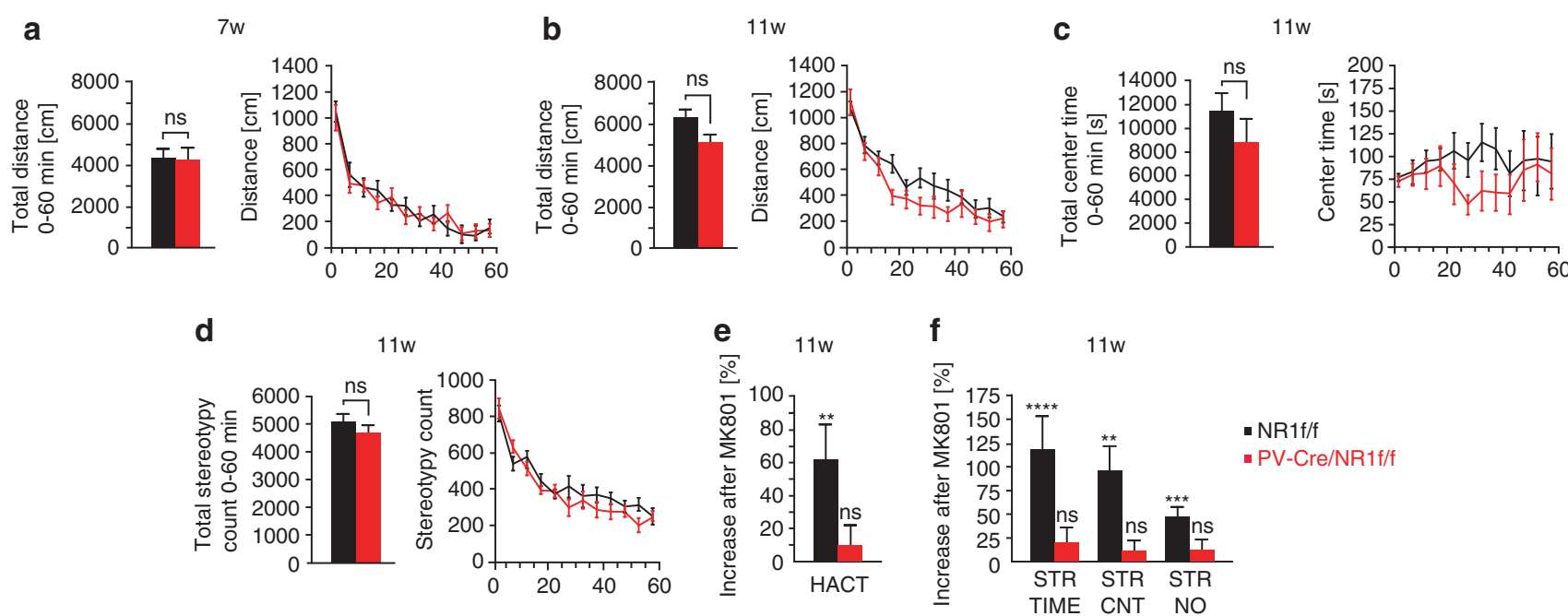

Figure 4 Parvalbumin (PV)-Cre/NR1f/f mice display no behavioral changes in the open field but reduced sensitivity to pharmacological NMDA receptor (NMDAR) treatment. (a) Total distance traveled over 60 min in the open field of PV-Cre/ NR1f/f and control mice at 7 weeks. (b-d) Open field behavior over $60 \mathrm{~min}$ at 11 weeks. (b) Total distance traveled over $60 \mathrm{~min}$ in the open field of PV-Cre/NR1f/f and control mice at 11 weeks. (c) No significant difference in the time spent in the center of the field between the genotypes. (d) PV-Cre/NR1f/f mice do not display stereotypy behavior. (e) MK-801 treatment induces significant increase in horizontal activity only in control mice. (f) MK-801 treatment results in marked increase in stereotypy behavior only in control mice. HACT, horizontal activity; STR TIME, stereotypy time; STR CNT, stereotypy count; STR NO, stereotypy numbers; ns $P>0.05,{ }^{*} P<0.01,{ }^{*} * P<0.001$, ${ }^{*} * * P<0.0001$; error bars, mean \pm s.e.m.

a
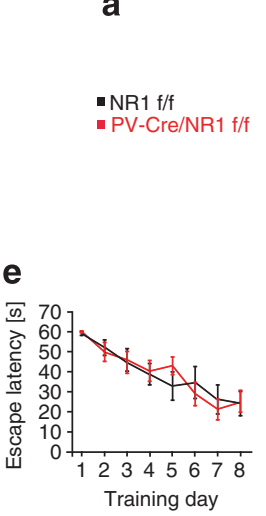

b
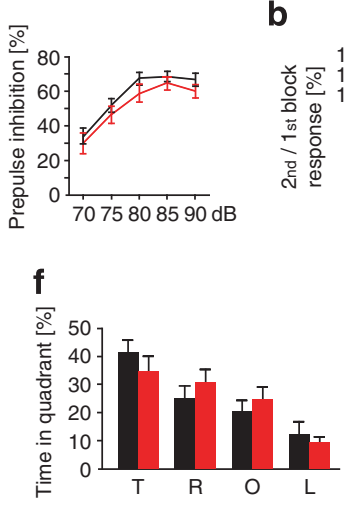

C
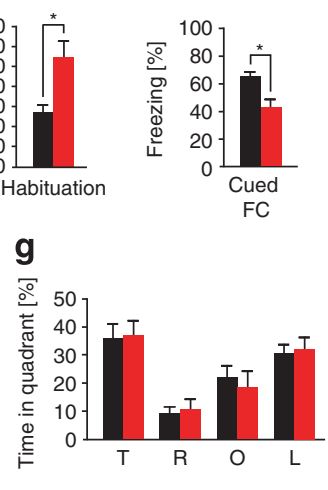

d

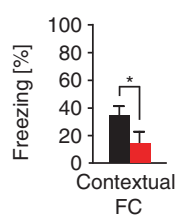

h

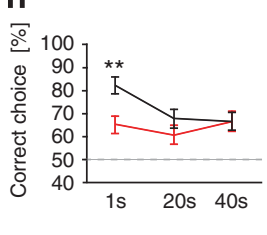

Figure 5 Loss of NMDA receptor (NMDAR) in parvalbumin (PV) interneurons results in selective cognitive disruptions. (a) PV-Cre/NR1f/f mice show no deficiency in sensorimotor gating as measured by prepulse inhibition (PPI). (b) PV-Cre/NR1f/f display deficiencies in habituation. (c, d), PV-Cre/NR1f/f mice exhibit impaired freezing behavior both to a tone-dependent (cued) and a contextual version of fear conditioning. (e) PV-Cre/NR1f/f mice perform similarly to control mice during training in a hidden platform version of water maze. (f) Time spent (\%) in each quadrant during the water maze probe trial. There is no significant difference between the genotypes. (g) Time spent (\%) in each quadrant during the water maze probe trial after reversal training. There is no significant difference between the genotypes. (h) PV-Cre/NR1f/f mice perform at a similar accuracy levels independent of working memory load in the discrete paired-trial variable-delay T-maze task. PPI, prepulse inhibition; FC, fear conditioning; T, target quadrant; R, right quadrant; $\mathrm{O}$, opposite quadrant; L, left quadrant. ${ }^{*} P<0.05$, ${ }^{*} P<0.01$; error bars, mean \pm s.e.m. See also Supplementary Figure 5.

unaffected first-degree relatives. Interneurons are considered to be important for phase-dependent inhibition and circuit oscillations that in turn regulate information processing. Our evaluation of PPI in PVCre/NR1f/f mice compared with control mice did not reveal any significant changes in PPI over several prepulse intensities (70-90 dB) (Figure 5a, $n=17$ control mice, 15 PV-Cre/NR1f/f mice). Importantly, the startle response was not different between control mice and PV-Cre/NR1f/f mice (Supplementary Figures 5a and b), thereby excluding sensorimotor defects.

Loss of NMDAR in PV cells results in selective cognitive disruptions

Several studies have analyzed the role of NMDAR in pyramidal neurons during learning and memory by pharmacological NMDAR inhibition ${ }^{43}$ or with genetic 
approaches. $^{23,44}$ The contribution of interneurons to learning and memory processes has been proposed ${ }^{45}$ but not genetically evaluated, and interneurons display similar NMDAR-dependent electrophysiological properties as pyramidal neurons. ${ }^{46}$ Habituation is considered to be one of the simplest forms of learning and is disrupted by NMDAR antagonists. ${ }^{41,47}$ Accordingly, we observed a significant reduction in acoustic startle habituation in PV-Cre/NR1f/f mice, supporting the NMDAR-dependent contribution of PV interneurons to learning and memory (Figure 5b and Supplementary Figure 5c).

To investigate the role of NMDAR in PV cells for associative learning processes, we exposed mice to cued and contextual fear conditioning paradigms ( $n=5$ per genotype). Mice were exposed to a neutral conditioned stimulus (sound) in a novel environment paired with an aversive foot shock as the unconditioned stimulus. PV-Cre/NR1f/f mice exhibited impaired associative learning both in a tone-dependent trial $24 \mathrm{~h}$ later $(P<0.05$, Mann-Whitney test; Figure $5 \mathrm{c})$ and a contextual version of the test $(P<0.05$, Mann-Whitney test; Figure 5d).

To evaluate spatial reference memory, we used a water maze task with a fixed hidden platform consisting of two trials per day with a 5-min intratrial interval. Infusion of NMDAR antagonists has revealed a critical role for NMDAR during learning and memory for spatial reference memory, ${ }^{43}$ although the specific contribution of NMDAR on interneurons has not been addressed. The rate of learning in the water maze task over 8 days was not impaired in PV-Cre/ NR1f/f mice compared with controls (Figure 5e; escape latency, $P=0.8794$, two-way analysis of variance; $n=8$ control and $11 \mathrm{PV}-\mathrm{Cre} / \mathrm{NR} 1 \mathrm{f} / \mathrm{f}$ mice). The probe trial on day 9 did not reveal significant differences in target quadrant preference compared with control mice $(P=0.33$ for target quadrant, unpaired $t$-test; Figure $5 \mathrm{f}$ ). To address potential impairments in reversal learning, we introduced the hidden platform in a different quadrant and continued the learning paradigm for 4 days. Learning rates for the new platform location over 4 days were not significantly different between genotypes $(P=0.513$, two-way analysis of variance) and a probe trial on the fifth day did not reveal target quadrant preference differences between genotypes $(P=0.9$ for target quadrant, unpaired $t$-test; Figure $5 \mathrm{~g}$ ).

Working memory deficits in schizophrenic patients, ${ }^{48-50}$ and computational models implicate NMDAR as a critical component of working memory ${ }^{51}$ with inhibition being important in shaping the time-dependent firing of pyramidal cells in neocortical circuits during cognitive tasks. ${ }^{52}$ Further, NMDAR antagonists disrupt working memory in humans ${ }^{53}$ and rodents. ${ }^{54}$ We assessed working memory in a T-maze (the discrete paired-trial variabledelay T-maze task; ${ }^{30} n=10$ control and nine PV-Cre/ NR1f/f mice). Both genotypes behaved similarly during the 10 days of training (Supplementary Figure 5d). After training, we performed 10 trials per mouse

each day with varying intratrial intervals, to evaluate effects of working memory load on performance. The PV-Cre/NR1f/f mice performed similarly to control mice at 20- and 40-s intratrial intervals (Figure 5h). In contrast, the 1-s intratrial interval revealed a marked difference between genotypes, with PV-Cre/NR1f/f mice remaining at $65 \%$ accuracy whereas control mice were significantly better with $82 \%$ accuracy $(P<0.01$; Figure 5h). PV-Cre/NR1f/f mice therefore performed at similar accuracy levels independent of working memory load.

\section{Discussion}

We have investigated the in vivo function of NMDAR specifically in FS-PV interneurons in regulating cortical brain rhythms and cognitive functions (Supplementary Table 1). This work is based on a longstanding hypothesis connecting PV interneuron dysfunction, NMDAR hypofunction and disturbances in brain rhythms associated with cognitive tasks/functions. We find that NMDAR signaling in FS-PV interneurons is critical for the regulation of gamma oscillations during baseline conditions as well as for gamma rhythm induction. The data we present on optogenetic drive in the superficial cortical layers are specific to FS-PV interneurons, as PV-expressing cells in these laminae are only FS interneurons. That said, PV-expressing neurons are present throughout the brain. One alternative cell type that could impact our findings is PV-expressing thalamic neurons, which typically project to the granular layers in cortex. There is correlative (neurophysiological), causal (optogenetic) and computational (modeling) evidence that neocortical gamma oscillations depend crucially on local FS interneurons, but these studies also suggest that the tonic level of excitation to the neocortical circuit is a key. As such, alternations in these thalamic neurons could have impacted, for example, our baseline data.

The inability of the cortical network to induce additional gamma oscillations by direct activation of FS-PV interneurons might indicate an impairment of network flexibility. The results suggest that PV-Cre/ NR1f/f mice exhibit spontaneous and evoked network abnormalities similar to those observed after low does administration of NMDAR antagonists. ${ }^{13}$ This is similar to findings in psychiatric patients, who display aberrant recruitment of cortical circuits and diminished evoked gamma rhythm in response to cognitive and sensory tasks. ${ }^{55}$ The reduced gammaband activity after NMDAR antagonist treatment in PV-Cre/NR1f/f mice supports the hypothesis that FSPV interneurons are an important target for pharmacological NMDAR blockade associated with altered gamma rhythms, ${ }^{13,56}$ consistent with our computational model of the PV-Cre/NR1f/f cortical circuit.

We have further found a dissociation between the requirement for NMDAR in FS-PV interneurons during baseline behavior versus demanding cognitive tasks. Although the small age-dependent effects in the 
open field may be of interest in light of behavioral changes associated with transitions from adolescence to adulthood, our results suggest a subtle behavioral effect at most of NMDAR deficiency in PV interneuron in the unchallenged state. This finding is in contrast to the phenotypes of hyperlocomotion and stereotypical behaviors in mice with general NMDAR hypofunction. ${ }^{31,32}$

Working memory includes executive components such as goal maintenance and interference control. ${ }^{57}$ It is difficult to establish the explicit role of executive components in rodent working memory tasks, and in addition, several cognitive processes and memory systems may be used in conjunction in the tasks. ${ }^{57}$ Although the selective working memory deficit in performance of the PV-Cre/NR1f/f mice at short delays in the discrete paired-trial variable-delay T-maze task most likely represent a complex network deficit, these data suggest that activation of NMDAR on PV interneurons is required for rapid initiation of the working memory encoding phase. In contrast, PVCre/NR1f/f mice displayed intact spatial reference memory, as measured in the Morris water maze task. In line with this, mice with deletion of the $\alpha$-amino-3hydroxy-5-methyl-4-isoxazolepropionic acid (AMPA) receptor GluR-A specifically in PV-expressing interneurons ${ }^{58}$ do not show impaired spatial reference memory, but have reduced kainate-induced gamma oscillations in hippocampal slices. Our results point to a specific role for NMDAR in PV interneurons in memory tasks involving the neocortex, amygdala and hippocampus. A potential unifying feature of these forms of memory is their dependence on intact gamma rhythms, which are enhanced during exposure to novel environments, ${ }^{59}$ but might have a lesser role in long-term learning in the water-maze task or the longer intratrial intervals in the T-maze task.

Evidence supporting the important role of NMDAR in interneurons has recently been provided through genetic deletion of NMDAR in a mixed population of GABAergic interneurons, including PV interneurons. ${ }^{60}$ This deletion results in a wide range of behavioral phenotypes, including noveltyinduced hyperlocomotion, PPI deficits and anxietylike effects, which were not observed in PV-Cre/ NR1f/f mice. Our model of a targeted adolescent disruption of NMDAR transmission specifically in PV interneurons provides a specific framework for understanding the role of NMDA transmission in PV interneurons for oscillatory activities in neuronal ensembles.

An important question is to what extent the observed circuit deficits depend on specific NMDAR-related activities, or represent a general hypoexcitability of PV interneurons conferred by diminished glutamatergic drive. It has been shown that reduced excitatory recruitment of PV interneurons through loss of $\alpha$-amino-3-hydroxy-5-methyl-4isoxazolepropionic acid (AMPA) receptors affects ongoing and induced gamma oscillations in hippocampal slices, ${ }^{58}$ with corresponding deficits in work- ing memory but not spatial reference memory. Our in vivo physiological, behavioral studies and computational modeling suggest that such deficits, found in our mouse model as well, could reflect a general decrease in excitability of PV interneurons.

There is direct evidence for NMDAR hypofunction in psychiatric patients. ${ }^{61}$ Additionally, human genetic data for schizophrenia-associated genes have implicated the NMDAR signaling pathway and disruption of the neuregulin-1/ErbB4 pathway. ${ }^{62-64}$ PV interneurons express ErbB4 protein ${ }^{65}$ and the neuregulin-1/ ErbB4 pathway has a role in gamma oscillations. ${ }^{66}$ However, we emphasize that the PV-Cre/NR1f/f mouse does not represent a model of schizophrenia, but rather contributes to the investigation of possibly one dimension of schizophrenia that includes cognitive defects. Further, under even the best circumstances, a mouse model is limited in its ability to recapitulate all complex cognitive dimensions that have evolved in humans.

\section{Conflict of interest}

The authors declare no conflict of interest.

\section{Acknowledgments}

We thank Tracey Petryshen, Erin Berry, Mike Lewis, Nadine Joseph and Ji-Song Guan for their valuable advice on behavioral assays. MC is supported by a NARSAD Young Investigator Award. MC and KM are supported by Knut and Alice Wallenbergs Foundation.

\section{References}

1 Jones RS, Buhl EH. Basket-like interneurones in layer II of the entorhinal cortex exhibit a powerful NMDA-mediated synaptic excitation. Neurosci Lett 1993; 149: 35-39.

2 Buzsaki G, Leung LW, Vanderwolf CH. Cellular bases of hippocampal EEG in the behaving rat. Brain Res 1983; 287: 139-171.

3 Traub RD, Whittington MA, Stanford IM, Jefferys JG. A mechanism for generation of long-range synchronous fast oscillations in the cortex. Nature 1996; 383: 621-624.

4 Cardin JA, Carlen M, Meletis K, Knoblich U, Zhang F, Deisseroth K et al. Driving fast-spiking cells induces gamma rhythm and controls sensory responses. Nature 2009; 459: 663-667.

5 Fries P. Neuronal gamma-band synchronization as a fundamental process in cortical computation. Annu Rev Neurosci 2009; 32: 209-224.

6 Sohal VS, Zhang F, Yizhar O, Deisseroth K. Parvalbumin neurons and gamma rhythms enhance cortical circuit performance. Nature 2009; 459: 698-702.

7 Uhlhaas PJ, Haenschel C, Nikolic D, Singer W. The role of oscillations and synchrony in cortical networks and their putative relevance for the pathophysiology of schizophrenia. Schizophr Bull 2008; 34: 927-943.

8 Lewis DA, Hashimoto T, Volk DW. Cortical inhibitory neurons and schizophrenia. Nat Rev Neurosci 2005; 6: 312-324.

9 Cunningham MO, Hunt J, Middleton S, LeBeau FE, Gillies MJ, Davies CH et al. Region-specific reduction in entorhinal gamma oscillations and parvalbumin-immunoreactive neurons in animal models of psychiatric illness. J Neurosci 2006; 26: 2767-2776.

10 Kehrer C, Dugladze T, Maziashvili N, Wojtowicz A, Schmitz D, Heinemann U et al. Increased inhibitory input to CA1 pyramidal cells alters hippocampal gamma frequency oscillations in 
the MK-801 model of acute psychosis. Neurobiol Dis 2007; 25: $545-552$.

11 Sagratella S, Pezzola A, Popoli P, Scotti de Carolis AS. Different capability of N-methyl-D-aspartate antagonists to elicit EEG and behavioural phencyclidine-like effects in rats. Psychopharmacology (Berl) 1992; 109: 277-282.

12 Ma J, Leung LS. Relation between hippocampal gamma waves and behavioral disturbances induced by phencyclidine and methamphetamine. Behav Brain Res 2000; 111: 1-11.

13 Hakami T, Jones NC, Tolmacheva EA, Gaudias J, Chaumont J, Salzberg $\mathrm{M}$ et al. NMDA receptor hypofunction leads to generalized and persistent aberrant gamma oscillations independent of hyperlocomotion and the state of consciousness. PLoS One 2009; 4: e6755.

14 Ehrlichman RS, Gandal MJ, Maxwell CR, Lazarewicz MT, Finkel LH, Contreras D et al. N-methyl-d-aspartic acid receptor antagonist-induced frequency oscillations in mice recreate pattern of electrophysiological deficits in schizophrenia. Neuroscience 2009; 158: $705-712$.

15 Pinault D. N-methyl d-aspartate receptor antagonists ketamine and MK-801 induce wake-related aberrant gamma oscillations in the rat neocortex. Biol Psychiatry 2008; 63: 730-735.

16 Javitt DC, Zukin SR. Recent advances in the phencyclidine model of schizophrenia. Am J Psychiatry 1991; 148: 1301-1308.

17 Coyle JT, Tsai G, Goff D. Converging evidence of NMDA receptor hypofunction in the pathophysiology of schizophrenia. Ann NY Acad Sci 2003; 1003: 318-327.

18 Krystal JH, Anand A, Moghaddam B. Effects of NMDA receptor antagonists: implications for the pathophysiology of schizophrenia. Arch Gen Psychiatry 2002; 59: 663-664.

19 Saykin AJ, Gur RC, Gur RE, Mozley PD, Mozley LH, Resnick SM et al. Neuropsychological function in schizophrenia. Selective impairment in memory and learning. Arch Gen Psychiatry 1991; 48: $618-624$

20 Cho RY, Konecky RO, Carter CS. Impairments in frontal cortical gamma synchrony and cognitive control in schizophrenia. Proc Natl Acad Sci USA 2006; 103: 19878-19883.

21 Kwon JS, O’Donnell BF, Wallenstein GV, Greene RW, Hirayasu Y, Nestor PG et al. Gamma frequency-range abnormalities to auditory stimulation in schizophrenia. Arch Gen Psychiatry 1999; 56: 1001-1005.

22 Hippenmeyer S, Vrieseling E, Sigrist M, Portmann T, Laengle C, Ladle DR et al. A developmental switch in the response of DRG neurons to ETS transcription factor signaling. PLoS Biol 2005; 3: e159.

23 Tsien JZ, Huerta PT, Tonegawa S. The essential role of hippocampal CA1 NMDA receptor-dependent synaptic plasticity in spatial memory. Cell 1996; 87: 1327-1338.

24 Srinivas S, Watanabe T, Lin CS, William CM, Tanabe Y, Jessell TM et al. Cre reporter strains produced by targeted insertion of EYFP and ECFP into the ROSA26 locus. BMC Dev Biol 2001; 1: 4.

25 Ryu J, Futai K, Feliu M, Weinberg R, Sheng M. Constitutively active Rap2 transgenic mice display fewer dendritic spines, reduced extracellular signal-regulated kinase signaling, enhanced long-term depression, and impaired spatial learning and fear extinction. I Neurosci 2008; 28: 8178-8188.

26 Hung AY, Futai K, Sala C, Valtschanoff JG, Ryu J, Woodworth MA et al. Smaller dendritic spines, weaker synaptic transmission, but enhanced spatial learning in mice lacking Shank1. J Neurosci 2008; 28: 1697-1708.

27 Gasser T, Bacher P, Mocks J. Transformations towards the normal distribution of broad band spectral parameters of the EEG. Electroencephalogr Clin Neurophysiol 1982; 53: 119-124.

28 Cardin JA, Palmer LA, Contreras D. Stimulus-dependent gamma $(30-50 \mathrm{~Hz})$ oscillations in simple and complex fast rhythmic bursting cells in primary visual cortex. J Neurosci 2005; 25: 5339-5350.

29 Joho RH, Ho CS, Marks GA. Increased gamma- and decreased delta-oscillations in a mouse deficient for a potassium channel expressed in fast-spiking interneurons. J Neurophysiol 1999; 82: 1855-1864.

30 Aultman JM, Moghaddam B. Distinct contributions of glutamate and dopamine receptors to temporal aspects of rodent working memory using a clinically relevant task. Psychopharmacology (Berl) 2001; 153: 353-364

31 Mohn AR, Gainetdinov RR, Caron MG, Koller BH. Mice with reduced NMDA receptor expression display behaviors related to schizophrenia. Cell 1999; 98: 427-436.

32 Labrie V, Lipina T, Roder JC. Mice with reduced NMDA receptor glycine affinity model some of the negative and cognitive symptoms of schizophrenia. Psychopharmacology (Berl) 2008; 200: $217-230$

33 del Rio JA, de Lecea L, Ferrer I, Soriano E. The development of parvalbumin-immunoreactivity in the neocortex of the mouse. Brain Res Dev Brain Res 1994; 81: 247-259.

34 Fries P, Nikolic D, Singer W. The gamma cycle. Trends Neurosci 2007; 30: 309-316.

35 Bartos M, Vida I, Jonas P. Synaptic mechanisms of synchronized gamma oscillations in inhibitory interneuron networks. Nat Rev Neurosci 2007; 8: 45-56.

36 Andermann ML, Ritt J, Neimark MA, Moore CI. Neural correlates of vibrissa resonance; band-pass and somatotopic representation of high-frequency stimuli. Neuron 2004; 42: 451-463.

37 Swadlow HA. Efferent neurons and suspected interneurons in S-1 vibrissa cortex of the awake rabbit: receptive fields and axonal properties. J Neurophysiol 1989; 62: 288-308.

38 Andermann ML, Moore CI. A somatotopic map of vibrissa motion direction within a barrel column. Nat Neurosci 2006; 9: 543-551.

39 Javitt DC, Doneshka P, Zylberman I, Ritter W, Vaughan Jr HG. Impairment of early cortical processing in schizophrenia: an event-related potential confirmation study. Biol Psychiatry 1993; 33: $513-519$.

40 Vierling-Claassen D, Siekmeier P, Stufflebeam S, Kopell N Modeling GABA alterations in schizophrenia: a link between impaired inhibition and altered gamma and beta range auditory entrainment. J Neurophysiol 2008; 99: 2656-2671.

41 Braff DL, Geyer MA. Sensorimotor gating and schizophrenia. Human and animal model studies. Arch Gen Psychiatry 1990; 47: 181-188.

42 Turetsky BI, Calkins ME, Light GA, Olincy A, Radant AD, Swerdlow NR. Neurophysiological endophenotypes of schizophrenia: the viability of selected candidate measures. Schizophr Bull 2007; 33: 69-94.

43 Morris RG, Anderson E, Lynch GS, Baudry M. Selective impairment of learning and blockade of long-term potentiation by an Nmethyl-D-aspartate receptor antagonist, AP5. Nature 1986; 319 774-776.

44 Nakazawa K, Quirk MC, Chitwood RA, Watanabe M, Yeckel MF, Sun LD et al. Requirement for hippocampal CA3 NMDA receptors in associative memory recall. Science 2002; 297: 211-218.

45 Paulsen O, Moser EI. A model of hippocampal memory encoding and retrieval: GABAergic control of synaptic plasticity. Trends Neurosci 1998; 21: 273-278.

46 Lamsa K, Heeroma JH, Kullmann DM. Hebbian LTP in feedforward inhibitory interneurons and the temporal fidelity of input discrimination. Nat Neurosci 2005; 8: 916-924.

47 Klamer D, Palsson E, Revesz A, Engel JA, Svensson L. Habituation of acoustic startle is disrupted by psychotomimetic drugs: differential dependence on dopaminergic and nitric oxide modulatory mechanisms. Psychopharmacology (Berl) 2004; 176: $440-450$.

48 Goldman-Rakic PS. The physiological approach: functional architecture of working memory and disordered cognition in schizophrenia. Biol Psychiatry 1999; 46: 650-661.

49 Wexler BE, Stevens AA, Bowers AA, Sernyak MJ, Goldman-Rakic PS. Word and tone working memory deficits in schizophrenia Arch Gen Psychiatry 1998; 55: 1093-1096.

50 Forbes NF, Carrick LA, McIntosh AM, Lawrie SM. Working memory in schizophrenia: a meta-analysis. Psychol Med 2009; 39: 889-905.

51 Lisman JE, Fellous JM, Wang XJ. A role for NMDA-receptor channels in working memory. Nat Neurosci 1998; 1: 273-275.

52 Constantinidis C, Williams GV, Goldman-Rakic PS. A role for inhibition in shaping the temporal flow of information in prefrontal cortex. Nat Neurosci 2002; 5: 175-180.

53 Krystal JH, Karper LP, Seibyl JP, Freeman GK, Delaney R, Bremner JD et al. Subanesthetic effects of the noncompetitive NMDA 
antagonist, ketamine, in humans. Psychotomimetic, perceptual, cognitive, and neuroendocrine responses. Arch Gen Psychiatry 1994; 51: 199-214.

54 Tonkiss J, Rawlins JN. The competitive NMDA antagonist AP5, but not the non-competitive antagonist MK801, induces a delayrelated impairment in spatial working memory in rats. Exp Brain Res 1991; 85: 349-358.

55 Basar-Eroglu C, Brand A, Hildebrandt H, Karolina Kedzior K, Mathes B, Schmiedt C. Working memory related gamma oscillations in schizophrenia patients. Int J Psychophysiol 2007; 64: 39-45.

56 Homayoun H, Moghaddam B. NMDA receptor hypofunction produces opposite effects on prefrontal cortex interneurons and pyramidal neurons. J Neurosci 2007; 27: 11496-11500.

57 Arguello PA, Gogos JA. Cognition in mouse models of schizophrenia susceptibility genes. Schizophr Bull 2010; 36: 289-300.

58 Fuchs EC, Zivkovic AR, Cunningham MO, Middleton S, Lebeau $\mathrm{FE}$, Bannerman DM et al. Recruitment of parvalbumin-positive interneurons determines hippocampal function and associated behavior. Neuron 2007; 53: 591-604.

59 Nitz D, McNaughton B. Differential modulation of CA1 and dentate gyrus interneurons during exploration of novel environments. J Neurophysiol 2004; 91: 863-872.

60 Belforte JE, Zsiros V, Sklar ER, Jiang Z, Yu G, Li Y et al. Postnatal NMDA receptor ablation in corticolimbic interneurons confers schizophrenia-like phenotypes. Nat Neurosci 2010; 13: 76-83.

61 Pilowsky LS, Bressan RA, Stone JM, Erlandsson K, Mulligan RS, Krystal JH et al. First in vivo evidence of an NMDA receptor deficit in medication-free schizophrenic patients. Mol Psychiatry 2006; 11: 118-119.

62 Stefansson H, Sigurdsson E, Steinthorsdottir V, Bjornsdottir S, Sigmundsson T, Ghosh S et al. Neuregulin 1 and susceptibility to schizophrenia. Am J Hum Genet 2002; 71: 877-892.

63 Hahn CG, Wang HY, Cho DS, Talbot K, Gur RE, Berrettini WH et al. Altered neuregulin 1-erbB4 signaling contributes to NMDA receptor hypofunction in schizophrenia. Nat Med 2006; 12: 824-828.

64 Lau CG, Zukin RS. NMDA receptor trafficking in synaptic plasticity and neuropsychiatric disorders. Nat Rev Neurosci 2007; 8: 413-426.

65 Neddens J, Buonanno A. Selective populations of hippocampal interneurons express ErbB4 and their number and distribution is altered in ErbB4 knockout mice. Hippocampus 2010; 20: 724-744.

66 Fisahn A, Neddens J, Yan L, Buonanno A. Neuregulin-1 modulates hippocampal gamma oscillations: implications for schizophrenia. Cereb Cortex 2009; 19: 612-618.

(c) This work is licensed under the Creative Commons Attribution-NonCommercialNo Derivative Works 3.0 Unported License. To view a copy of this license, visit http://creativecommons. org/licenses/by-nc-nd/3.0/

Supplementary Information accompanies the paper on the Molecular Psychiatry website (http://www.nature.com/mp) 